International Mathematical Forum, 2, 2007, no. 30, 1457 - 1468

\title{
Invariance Properties of the Negative Binomial Lévy Process and Stochastic Self-similarity
}

\author{
Tomasz J. Kozubowski \\ Department of Mathematics \& Statistics \\ University of Nevada at Reno \\ Reno, NV 89557, USA \\ tkozubow@unr.edu \\ Krzysztof Podgórski \\ Department of Mathematical Sciences \\ Indiana University-Purdue University Indianapolis \\ Indianapolis, IN 46202-3216, USA \\ kpodgorski@math.iupui.edu
}

\begin{abstract}
We study the concept of self-similarity with respect to stochastic time change. The negative binomial process (NBP) is an example of a family of random time transformations with respect to which stochastic self-similarity holds for certain stochastic processes. These processes include gamma process, geometric stable processes, Laplace motion, and fractional Laplace motion. We derive invariance properties of the NBP with respect to random time deformations in connection with stochastic self-similarity. In particular, we obtain more general classes of processes that exhibit stochastic self-similarity properties. As an application, our results lead to approximations of the gamma process via the NBP and simulation algorithms for both processes.
\end{abstract}

Mathematics Subject Classification: 60E07, 60G18, 60G51, 60G55, $60 \mathrm{~J} 75$

Keywords: Compound Poisson process, Cox process, Discrete Lévy process, Doubly stochastic Poisson process, Fractional Laplace motion, GammaPoisson process, Gamma process, Geometric sum, Geometric distribution, Infinite divisibility, Point process, Random stability, Subordination, Self similarity, Simulation 


\section{Introduction}

A stochastic process $X(t)$ is called self-similar with index $H$ if for all $c>$ 0 we have $X(c \cdot) \stackrel{\mathrm{d}}{=} c^{H} X(\cdot)$, where $\stackrel{\mathrm{d}}{=}$ stands for the equality of the finite dimensional distributions (see, e.g., [5]). This property shows the equivalence between argument scale change (time scale change) and the (value) space scale change. Many phenomena, such as stock market variability (see, e.g., [8]), are characterized by intrinsic time (operational time) that is stochastic when related to the objective time. Therefore, it is relevant to consider a random time change, understood as subordination to a family of non-negative, nondecreasing stochastic processes indexed by $c \geq 1$, whose expectations are given by $c t$. It was noted in [10] that the family of negative binomial Lévy processes with drift provide random time transformations with respect to which some stochastic processes have this form of self-similarity. In this work we investigate this further. In Section 2 we review the notion of stochastic self-similarity, which parallels the classical self-similarity, and study its relation to the negative binomial (NB) and gamma Lévy processes. In Section 3 we discuss more general classes of processes that exhibit stochastic self-similarity properties. Our results lead to approximations of the gamma process via the negative binomial process (NBP) and a new simulation algorithm of the gamma process, discussed in Section 4. For the convenience of the reader, we collect basic facts about the NBP in an appendix.

\section{Stochastic self-similarity and invariance prop- erties of the NBP}

Let us start with a formal definition of self-similarity with respect stochastic time transformations. Let $\mathcal{T}=\left\{T_{c}(t), t \geq 0\right\}, c \geq 1$, be a family of random time changes with $\mathbb{E} T_{c}(t)=c t$. Following [10], we say that a process $X(t), t \geq 0$, is stochastically self-similar with index $H$ with respect to $\mathcal{T}$ if $X\left(T_{c}(\cdot)\right) \stackrel{\mathrm{d}}{=} c^{H} X(\cdot)$ for each $c \geq 1$. Despite this apparently non-restrictive definition, the only currently known non-trivial examples of stochastic selfsimilar processes are those with respect to the NB time change (and their generalizations presented in Section 3).

Remark 2.1. This notion of stochastic self-similarity, which involves stochastic renormalization in time, is apparently unrelated to that considered in [7] and [21], which involves stochastic renormalization in space.

Recall that a negative binomial Lévy process (NBP) is a continuous time process $\left\{N B_{p}(t), t \geq 0\right\}$ starting at zero with independent and homogenous increments, where the lag- $t$ increments have a NB distribution with parameter 
$p \in(0,1)$, given by the characteristic function $(\mathrm{ChF})$

$$
\phi_{N B_{p}(t)}(u)=\left(\frac{p}{1-(1-p) e^{i u}}\right)^{t}, u \in \mathbb{R},
$$

and the probability mass function (PMF)

$$
\mathbb{P}_{N B_{p}(t)}(k)=\left(\begin{array}{c}
t+k-1 \\
k
\end{array}\right) p^{t}(1-p)^{k}, \quad k=0,1,2, \ldots,
$$

see [11]. Let

$$
\mathcal{T}=\left\{T_{c}(t)=t+N B_{p}(t), t \geq 0\right\}, c \geq 1,
$$

where $N B_{p}(t)$ is a NBP with parameter $p=1 / c$, be a family of NB processes with drift. Since $E T_{c}(t)=c t,(3)$ is a family of stochastic time changes, referred to as the NB time changes. The following result appeared in [10].

Proposition 2.2. Let $\Gamma(t), t \geq 0$, be a standard gamma Lev́y process. Then $\Gamma(t)$ is stochastically self-similar with index $H=1$ with respect to the family (3) of NB time changes.

The family (3) possess an interesting group property, given in the next result.

Proposition 2.3. If $T_{c_{1}}(\cdot)$ and $T_{c_{2}}(\cdot)$ are independent members of the family $\mathcal{T}$ given by (3), then

$$
T_{c_{1}} \circ T_{c_{2}}(\cdot) \stackrel{\mathrm{d}}{=} T_{c_{1} c_{2}}(\cdot)
$$

where o stands for the composition of two functions.

Proof. The ChF of $T_{c}(t)$ is given by $\phi_{c, t}(u)=e^{i t u}\left[c+(1-c) e^{i u}\right]^{-t}$. Since $T_{c_{1}} \circ T_{c_{2}}(\cdot)$ and $T_{c_{1} c_{2}}(\cdot)$ are both Lévy processes, it is enough to show that the $\mathrm{ChF}$ of $T_{c_{1}} \circ T_{c_{2}}(1)$ is given by

$$
\phi(u)=\frac{e^{i u}}{c_{1} c_{2}+\left(1-c_{1} c_{2}\right) e^{i u}} .
$$

Conditioning on $T_{c_{2}}(1)$ produces

$$
\begin{gathered}
\mathbb{E} \exp \left(i T_{c_{1}}\left(T_{c_{2}}(1)\right) u\right)=\sum_{k=1}^{\infty}\left(\frac{e^{i u}}{c_{1}+\left(1-c_{1}\right) e^{i u}}\right)^{k}\left(\frac{c_{2}-1}{c_{2}}\right)^{k} \frac{1}{c_{2}-1} \\
=\frac{1}{c_{2}-1} \frac{e^{i u}\left(c_{2}-1\right)}{c_{2}\left(c_{1}+\left(1-c_{1}\right) e^{i u}\right)-e^{i u}\left(c_{2}-1\right)}
\end{gathered}
$$

Since the last expression coincides with (5) the result follows. 
Another interesting invariance property is obtained when a NBP is subject to an independent NB random time change.

Proposition 2.4. Let $N B_{p}(\cdot)$ be a NBP with parameter $p$, independent of a random time change $T_{c}(\cdot)$ belonging to the family (3). Then $N B_{p} \circ T_{c}(\cdot)$ is again a NBP process with parameter $1 /(1+c(1 / p-1))$.

Proof. By Proposition 5.1 (see appendix), the process $N B_{p}(t)$ can be written as $N(\Gamma(t))$ where $N(\cdot)$ is a Poisson process with $\lambda=q / p$ and $\Gamma(t)$ is a standard gamma process. By Proposition $2.2, \Gamma\left(T_{c}(\cdot)\right) \stackrel{\mathrm{d}}{=} c \Gamma(\cdot)$, so that $\left.N B_{p} \circ T_{c}(\cdot)\right) \stackrel{\mathrm{d}}{=} N(c \Gamma(\cdot))=\tilde{N}(\Gamma(\cdot))$, where $\tilde{N}$ is a Poisson process with intensity $c q / p$. However, $c q / p=(1-\tilde{p}) / \tilde{p}$ when $\tilde{p}=1 /(1+c(1 / p-1))$.

Although the composition of negative binomial processes is not a group operation within the class of negative binomial processes, it leads to the following invariance relation.

Corollary 2.5. Let $N B_{p_{1}}, \widetilde{N B}_{p_{1}}$ and $N B_{p_{2}}$ be three independent $N B P$ s with parameters $p_{1}, p_{1}$, and $p_{2}$, respectively. Then the process

$$
N B_{p_{1}} \circ N B_{p_{2}}(\cdot)+\widetilde{N B}_{p_{1}}(\cdot)
$$

is also a NBP with parameter $\tilde{p}=p_{1} p_{2} /\left(p_{1} p_{2}+\left(1-p_{1}\right)\right)$.

Proof. As always in the case of Lévy processes it is enough to consider one dimensional distributions. By Proposition 2.4, we have

$$
\begin{aligned}
N B_{\tilde{p}}(1) & =N B_{p_{1}}\left(1+N B_{p_{2}}(1)\right) \\
& =N B_{p_{1}}\left(1+N B_{p_{2}}(1)\right)-N B_{p_{1}}\left(N B_{p_{2}}(1)\right)+N B_{p_{1}}\left(N B_{p_{2}}(1)\right) \\
& =\Delta N B_{p_{1}}(1)+N B_{p_{1}} \circ N B_{p_{2}}(1),
\end{aligned}
$$

where the last equality serves as the definition of $\Delta N B_{p_{1}}(1)$. Next, by the independence and homogeneity of the increments, for each $A, B \subset \mathbb{R}$ we have

$$
\begin{aligned}
\mathbb{P}\left(\Delta N B_{p_{1}}(1)\right. & \left.\in A, N B_{p_{1}} \circ N B_{p_{2}}(1) \in B\right) \\
& =\mathbb{E}\left(\mathbb{P}\left(\Delta N B_{p_{1}}(1) \in A, N B_{p_{1}} \circ N B_{p_{2}}(1) \in B \mid N B_{p_{2}}(1)\right)\right) \\
& =\mathbb{P}\left(N B_{p_{1}}(1) \in A\right) \cdot \mathbb{E}\left(\mathbb{P}\left(N B_{p_{1}}\left(N B_{p_{2}}(1)\right) \in B \mid N B_{p_{2}}(1)\right)\right) \\
& =\mathbb{P}\left(N B_{p_{1}}(1) \in A\right) \cdot \mathbb{P}\left(N B_{p_{1}} \circ N B_{p_{2}}(1) \in B\right),
\end{aligned}
$$

which shows that $N B_{\tilde{p}}(\cdot)$ has the representation (6).

The following stability property of the gamma process with respect to the composition with the NBP is used in Section 4, where it is applied to approximate the gamma process. It allows for a decomposition of a gamma process into two independent components, of which one is negligible while the other involves a standard gamma process evaluated only at integer values of $N B_{p}(t)$. 
Proposition 2.6. Let $\tilde{\Gamma}(\cdot)$ and $\Gamma(\cdot)$ be independent standard gamma processes, which are also independent of a negative binomial process $N B_{p}(\cdot)$. Then for each $p \in(0,1)$,

$$
\Gamma(\cdot) \stackrel{\mathrm{d}}{=} p \cdot \Gamma(\cdot)+p \cdot \tilde{\Gamma} \circ N B_{p}(\cdot) .
$$

Proof. By the stochastic self-similarity of the gamma process expressed in Proposition 2.2 , we have $\Gamma(\cdot) \stackrel{\mathrm{d}}{=} p \cdot \Gamma \circ T_{1 / p}(\cdot)$. Since the processes on both the sides of $(7)$ are Lévy, we just need to show that

$$
p \cdot \Gamma\left(1+N B_{p}(1)\right) \stackrel{\mathrm{d}}{=} p \cdot \Gamma(1)+p \cdot \tilde{\Gamma}\left(N B_{p}(1)\right) .
$$

This follows from the independence and homogeneity of increments of the gamma process, and the proof is similar to that of Corollary 2.5.

\section{A generalization}

The proof of Proposition 2.3 is based on the fact that the family of probability generating functions of geometric random variables forms a commutative group. More precisely, for $p \in(0,1)$ let $T_{1 / p}$ be a geometric random variable with mean $1 / p$ and the probability generating function (PGF)

$$
H_{p}(s)=\mathbb{E} s^{T_{1 / p}}=\frac{p s}{1-(1-p) s}, s \in[0,1) .
$$

One can easily check that for each $p_{1}, p_{2}$ in $(0,1)$ we have

$$
H_{p_{1}} \circ H_{p_{2}}(s)=H_{p_{1}}\left(H_{p_{2}}(s)\right)=H_{p_{2}}\left(H_{p_{1}}(s)\right)=H_{p_{1} p_{2}}(s), \quad s \in[0,1) .
$$

We can write the above property of the PGFs in the language of random variables: a geometric convolution of IID geometric random variables is again geometric, that is

$$
\sum_{k=1}^{T_{c_{1}}} T_{c_{2}, k} \stackrel{d}{=} T_{c_{1} c_{2}}, \quad c_{1}, c_{2} \in(1, \infty),
$$

where the $\left\{T_{c_{2}, k}\right\}$ are IID copies of the (geometric) variable $T_{c_{2}}$, independent of $T_{c_{1}}$.

This suggest the following generalization. Consider a family $\left\{N_{p}, p \in\right.$ $(0,1)\}$ of infinitely divisible random variables taking non-negative integer values, and define a class of processes

$$
\left\{T_{1 / p}(t)=t+N_{p}(t), t \geq 0\right\}, \quad p \in(0,1),
$$

denoting, as before, $T_{1 / p}=T_{1 / p}(1)$. Then we have the following analog of Proposition 2.3 with the same proof. 
Proposition 3.1. If (9) is a family of Lévy processes with drift and the PGFs corresponding to the variables $T_{1 / p}(1)$ form a commutative group, then the group property (4) holds.

Proof. Since both $T_{c_{1}} \circ T_{c_{2}}(\cdot)$ and $T_{c_{1} c_{2}}(\cdot)$ are Lévy processes, it is enough to show the equality in distribution for $t=1$. To this end, note that the PGF of $T_{c_{1}} \circ T_{c_{2}}(1)$ is

$$
\mathbb{E} s^{T_{c_{1}}\left(T_{c_{2}}(1)\right)}=\sum_{k=1}^{\infty}\left[G_{1 / c_{1}}(s)\right]^{k} \mathbb{P}\left(T_{c_{2}}(1)=k\right)=G_{1 / c_{2}}\left(G_{1 / c_{1}}(s)\right)=G_{1 / c_{1} c_{2}}(s),
$$

which is the PGF of $T_{c_{1} c_{2}}(1)$. This completes the proof.

Next, consider any family $\left\{N_{p}, p \in(0,1)\right\}$ of random variables taking nonnegative integer values such that the PGFs corresponding to the variables $N_{p}+1$ form a commutative group. For each $k \in \mathbb{N}$ let

$$
N_{p}^{(k)}=k N_{p}(1 / k), \quad p \in(0,1),
$$

where $N_{p}(1 / k)$ is the $1 / k$-lag increment of the Lévy process corresponding to $N_{p}$. These variables are again infinitely divisible, and lead to a class of Lévy processes $\left\{N_{p}^{(k)}(t), t \geq 0\right\}$, where $p \in(0,1)$ and $k \in \mathbb{N}$. For each fixed $k \in \mathbb{N}$, we have the corresponding class of processes with drift,

$$
\left\{T_{1 / p}^{(k)}(t)=t+N_{p}^{(k)}(t), t \geq 0\right\}, \quad p \in(0,1)
$$

and the PGFs $H_{p}^{(k)}(\cdot)$ corresponding to the variables $T_{1 / p}^{(k)}=T_{1 / p}^{(k)}(1), p \in(0,1)$, form a commutative group. That is, for each $p_{1}, p_{2} \in(0,1)$ we have

$$
H_{p_{1}}^{(k)} \circ H_{p_{2}}^{(k)}(s)=H_{p_{1}}^{(k)}\left(H_{p_{2}}^{(k)}(s)\right)=H_{p_{2}}^{(k)}\left(H_{p_{1}}^{(k)}(s)\right)=H_{p_{1} p_{2}}^{(k)}(s), \quad s \in[0,1) .
$$

Note that the distributions of $N_{p}^{(k)}$ and $T_{1 / p}^{(k)}$ are supported on the sets of integers $\{0, k, 2 k, \ldots\}$ and $\{1, k+1,2 k+1, \ldots\}$, respectively. In addition, whenever the expected values of $T_{1 / p}^{(k)}$ and $T_{1 / p}$ are finite, they coincide (and are equal to $1 / p$ in the geometric case).

We now derive a Lévy process that is stochastically self-similar with respect to the family $\left\{T_{c}(t), t \geq 0\right\}, c \geq 1$. Here, we shall assume the convergence $p N_{p} \stackrel{d}{\rightarrow} Z$ as $p$ approaches zero, where $Z$ is a non-negative random variable with the Laplace transform $\psi(\cdot)$. Observe that the group property of the processes $T_{c}$ leads to random stability of the distribution of $Z$. To see this, divide both sides of (8) by $c_{2}$ and let $c_{2}$ increase without bound. Since $T_{c_{2}} / c_{2}=$ $\left(N_{1 / c_{2}}-1\right) / c_{2}$ approaches $Z$, we obtain

$$
\sum_{k=1}^{T_{c}} Z_{k} \stackrel{d}{=} c Z, \quad c>1
$$


where the $\left\{Z_{k}\right\}$ are IID copies of $Z$. Writing the above in terms of Laplace transforms, we obtain $H_{c}(\psi(s))=\psi(c s), s \in[0,1)$, where $H_{c}(u)$ is the PGF of $T_{c}$. Equivalently, denoting $r=\psi(s)$, we have $H_{c}(r)=\psi\left(c \psi^{-1}(r)\right)$, a functional equation that arises in the theory of branching processes when $\mathbb{E} T_{c}=c$ (where $Z$ corresponds to the limiting distribution of the population size). (More details on this connection and relation of this equation to random stability can be found in [3].) Since $Z$ is a weak limit of infinitely divisible variables, it is itself infinitely divisible, and defines a Lévy process $\{Z(t), t \geq 1\}$, whose lag-1 increment has the same distribution as $Z$. Now, the stability property (11) implies that this process is stochastically self-similar (with index $H=1$ ) with respect to the family $\left\{T_{c}(t), t \geq 0\right\}, c \geq 1$. Indeed, we have

$$
Z\left(T_{c}(1)\right)=\sum_{k=1}^{T_{c}(1)}[Z(k)-Z(k-1)] \stackrel{d}{=} c Z(1)
$$

where $Z(k)-Z(k-1) \stackrel{d}{=} Z(1) \stackrel{d}{=} Z$, so that the (Lévy) processes $\left\{Z\left(T_{c}(t)\right), t \geq\right.$ $0\}$ and $\{c Z(t), t \geq 0\}$ have the same finite dimensional distributions.

Remark 3.2. Note that in the case of geometric variables $N_{p}$ the limiting distribution $Z$ is standard exponential with the Laplace transform $\psi(s)=$ $1 /(1+s)$. The stability properties of exponential variables with respect to geometric compounding were discussed by Arnold [1]. The corresponding family $\left\{N_{p}^{(k)}, p \in(0,1)\right\}$ consists on negative binomial variables re-scaled by $k$, that is $N_{p}^{(k)} \stackrel{d}{=} k N B_{p}(1 / k)$. Here, as $p$ approaches zero, for each integer $k \geq 1$ we have the convergence $p N_{p}^{(k)} \stackrel{d}{\rightarrow} Z^{(k)}$, where $Z^{(k)}$ is a non-negative random variable with the Laplace transform $[\psi(s k)]^{1 / k}$. This variable has the representation $Z^{(k)} \stackrel{d}{=} k Z(1 / k)$, where $Z(\cdot)$ is the marginal distribution of the Lévy process above, and, in the case of geometric $N_{p}$, has a gamma distribution with shape parameter $1 / k$ and scale parameter $k$ (so that its mean is equal to 1 ). This distribution admits the stability property (11), were the (random) number of terms in the summation is $T_{c_{1}}^{(k)}$ (see [3], and also [18, 19], where this distribution is referred to as Harris law). The Lévy process whose lag-1 increments have the same distribution as $Z^{(k)}$ are stochastically self-similar (with index $H=1)$ with respect to the family $\left\{T_{c}^{(k)}(t), t \geq 0\right\}, c \geq 1$.

Remark 3.3. Note that for any $\alpha \in(0,1]$ the random variable $Z_{\alpha}$ with the Laplace transform $\psi_{\alpha}(s)=\psi\left(s^{\alpha}\right)$, where $\psi(\cdot)$ is the Laplace transform of $Z$, is infinitely divisible. This follows from the stochastic representation $Z_{\alpha} \stackrel{d}{=}$ $Z^{1 / \alpha} S_{\alpha}$, where $S_{\alpha}$ is a stable subordinator - a positive random variable with the Laplace transform $\exp \left(-s^{\alpha}\right)$. Since both $Z$ and $S_{\alpha}$ are infinitely divisible, so is the mixture above (see [6], Property (e), XVII.4). Further, the variables 
$Z_{\alpha}$ have the stability property with respect to the same family as the $\left\{Z_{k}\right\}$,

$$
\sum_{k=1}^{T_{c}} Z_{\alpha, k} \stackrel{d}{=} c^{1 / \alpha} Z_{\alpha}, \quad p_{1} \in(0,1),
$$

where the $\left\{Z_{\alpha, k}\right\}$ are IID copies of $Z_{\alpha}$. This is most easily seen by expressing (12) in terms of the Laplace transforms, $H_{c}\left(\psi_{\alpha}(s)\right)=\psi_{\alpha}\left(s c^{1 / \alpha}\right)$. This implies that the corresponding Lévy process $\left\{Z_{\alpha}(t), t \geq 0\right\}$, whose lag-1 increments are distributed as $Z_{\alpha}$, is stochastically self-similar with index $H=1 / \alpha$ with respect to the family $\left\{T_{c}(t), t \geq 0\right\}, c \geq 1$. If $Z$ is exponential, then $Z_{\alpha}$ has a Mittag-Leffler distribution (see [16]), which belongs to a more general class of geometric stable laws (see, e.g., [12]). These are exponential scale mixtures of general stable laws.

Remark 3.4. It is easy to see that if $\{X(t), t \geq 0\}$ is any self-similar process with index $H$ and $\{Z(t), t \geq 0\}$ is an independent Lévy process that is stochastically self-similar with index $H^{\prime}$ with respect to a family $\mathcal{T}=\left\{T_{c}(t), t \geq 0\right\}_{c \geq 1}$, then the subordinated process $\{Y(t)=X(Z(t)), t \geq 0\}$ is again stochastically self-similar with index $H H^{\prime}$ with respect to the same family. When $X(t)$ is a Brownian motion and $Z(t)$ is a gamma process, we obtain the Laplace motion discussed in [9], which plays an important role in mathematical finance (where it is known as the variance-gamma process, see [4], [13], [14], [20]). Similarly, a process with correlated increments, termed a fractional Laplace motion by Kozubowski et al. [10] and Meerschaert et al. [15], is obtained by subordinating a fractional Brownian motion to the gamma process. Other stochastic self-similar processes can be obtained similarly by subordinating stable processes either to the gamma process or other Lévy process $Z(t)$ (or $\left.Z_{\alpha}(t)\right)$ discussed above.

\section{Approximating the gamma process}

The geometric distribution plays a similar role among discrete laws as the exponential distribution does among continuous distributions. Moreover, if $G_{p}$ is a geometric random variable then as $p$ decreases to zero the variables $p \cdot G_{p}$ converge in distribution to the standard exponential variable. Analogous relations hold between the Lévy motions generated by these distributions, i.e. between the NBP and the gamma process. We discuss such asymptotic schemes below. Our first result shows that rescaling a NBP leads to a gamma process in the limit.

Theorem 4.1. As $p \rightarrow 0$, the finite dimensional distributions of $p \cdot N B_{p}(\cdot)$ converge to those of the standard gamma process. 
Proof. For Lévy processes it is enough to demonstrate the convergence of one dimensional distributions. This can be best seen from the ChFs. Indeed, we have

$$
\lim _{p \rightarrow 0} \phi_{p N B_{p}(t)}(u)=\lim _{p \rightarrow 0}\left(\frac{1}{e^{i p u}-\left(e^{i p u}-1\right) / p}\right)^{t}=\left(\frac{1}{1-i u}\right)^{t},
$$

which is the $\mathrm{ChF}$ of $\Gamma(t)$.

Lemma 4.2. Let $L$ be a logarithmic random variable given by the PMF (17), and let $\left(E_{k}\right)_{k \in \mathbb{N}}$ be a sequence of standard exponential random variables, independent of $L$. Then the PDF and the ChF of the random sum $X=\sum_{k=1}^{L} E_{k}$ are given by

$$
f_{X}(x)=\frac{e^{-x}-e^{-p x}}{x \log p}, x>0
$$

and

$$
\phi_{X}(u)=\frac{\log (p-i u)-\log (1-i u)}{\log p}, u \in \mathbb{R}
$$

respectively.

Proof. Conditioning on $L$ leads to

$f_{X}(x)=-\frac{1}{\log p} \sum_{k=1}^{\infty} \frac{1}{(k-1) !} x^{k-1} \frac{q^{k}}{k} e^{-x}=-\frac{1}{\log p} \sum_{k=1}^{\infty} \frac{(x q)^{k}}{k !} \frac{1}{x} e^{-x}=\frac{e^{-x}-e^{-p x}}{x \log p}$.

The derivation of the $\mathrm{ChF}$ is similar.

The stability property of Proposition 2.6 has an equivalent compound negative binomial representation discussed in our next result.

Proposition 4.3. Let $E_{k}$ be a sequence of standard exponential random variables, independent of a standard gamma process $\Gamma(\cdot)$. Then

$$
\Gamma(\cdot) \stackrel{\mathrm{d}}{=} p \cdot \Gamma(\cdot)+p \sum_{k=1}^{N B_{p}(\cdot)} E_{k}
$$

Moreover, if $Y_{p}(\cdot)=\sum_{j=1}^{N(t)} X_{j}$ is a compound Poisson process, where $N(t)$ is a Poisson process with intensity $\lambda=-\log p$ and the $\left\{X_{j}\right\}$ are IID with distribution given in Lemma 4.2 and independent of the gamma process $\Gamma(\cdot)$, then $\Gamma(\cdot) \stackrel{d}{=} p\left(\Gamma(\cdot)+Y_{p}(\cdot)\right)$.

This leads to the following result. 
Theorem 4.4. In the notation of Proposition 4.3, as $p \rightarrow 0$, the finite dimensional distributions of the processes

$$
p \sum_{j=1}^{N(\cdot)} X_{j} \text { and } p \sum_{k=1}^{N B_{p}(\cdot)} E_{k}
$$

converge to those of a standard gamma process $\Gamma(\cdot)$.

Proof. Let $Z_{p}(\cdot)=p \Gamma(\cdot), X_{p}(\cdot)=p \sum_{i=1}^{N(\cdot)} X_{i}$, and $Y_{p}(\cdot)=\sum_{i=1}^{N B_{p}(\cdot)} E_{i}$. By Proposition $4.3, \Gamma_{p}(\cdot)=Z_{p}(\cdot)+X_{p}(\cdot)$ is a gamma process and $U_{p}(\cdot)=X_{p}(\cdot)-$ $\Gamma_{p}(\cdot)$ converges to zero (in the sense of convergence of finite dimensional distributions). Thus, the finite dimensional distributions of $X_{p}(\cdot)=U_{p}(\cdot)+\Gamma_{p}(\cdot)$ converge to the distributions of a gamma process. The same argument applies to $Y_{p}(\cdot)$.

The above results lead to an approximate method of simulating gamma processes using the NBP with a small value of $p$, which provides an alternative over the existing methods (see, e.g., $[2,17,20]$ ). The simplest approach is to use Theorem 4.1 and approximate the gamma process by a rescaled NBP. This leads to a process where the values of the jumps are multiplicities of $p$. Continuous values of jumps can be obtained using the second approximation provided by Theorem 4.4, along with one of the algorithms of simulating the NBP described in [11].

\section{Appendix}

Here we present different representations of the NBP, discussed in [11].

Proposition 5.1. Let $N B(t)$ be a stochastic process. The following conditions are equivalent:

(i) $N B_{p}(t)$ is a Lévy process corresponding to the geometric distribution given by the $P M F$

$$
\mathbb{P}(k ; p)=p(1-p)^{k}, k=0,1, \ldots
$$

(ii) $N B_{p}(t)$ is a Poisson process $\{N(t), t \geq 0\}$ with intensity $\lambda=(1-p) / p$ subordinated to a standard gamma process $\{\Gamma(t), t \geq 0\}$,

$$
N B(t)=N(\Gamma(t))
$$

(iii) $N B_{p}(t)$ is a compound Poisson process, that is

$$
N B_{p}(t)=X_{1}+\cdots+X_{N(t)}
$$


where $\{N(t), t \geq 0\}$ is a Poisson process with intensity $\lambda=-\log p$ and the $\left\{X_{i}\right\}$ have the logarithmic distribution given by the PMF

$$
\mathbb{P}(k ; p)=-\frac{(1-p)^{k}}{k \log p}, \quad k \in \mathbb{N} .
$$

It follows that the NBP is an integer valued, non-decreasing, and consequently a pure jump process, whose mean and variance are linear in $t$, $\mathbb{E} N B_{p}(t)=t \cdot(1-p) / p$ and $\operatorname{Var} N B_{p}(t)=t \cdot(1-q) / p^{2}$. Moreover, the ChF of the NBP admit the Lévy-Kchintchine representation

$$
\phi_{N B_{p}(t)}(u)=\exp \left(t \int\left(e^{i x u}-1\right) d \Lambda(x)\right)
$$

where the Lévy measure $\Lambda$ is discrete and given by $\Lambda=\sum_{k=1}^{\infty} \frac{(1-p)^{k}}{k} \delta_{\{k\}}$.

\section{References}

[1] B.C. Arnold, Some characterizations of the exponential distribution by geometric compounding, SIAM J. Appl. Math., 24(2) (1973), 242-244.

[2] L. Bondesson, On simulation from infinitely divisible distributions, $A d v$. Appl. Probab., 14 (1982), 855-869.

[3] J. Bunge, Composition semigroups and random stability, Ann. Probab., 24(3) (1996), 1476-1489.

[4] R. Cont and P. Tankov, Financial Modelling with Jump Processes, Chapman \& Hall/CRC, Boca Raton, 2004.

[5] P. Embrechts and M. Maejima, Selfsimilar Processes, Princeton University Press, Princeton and Oxford, 2002.

[6] V. Feller, Introduction to the Theory of Probability and its Applications, Vol. 2 (2nd ed.), John Wiley \& Sons, New York, 1971.

[7] V.K. Gupta and E.C. Waymire, Multiscaling properties of spatial rainfall in river flow distributions, J. Geophys. Res., 95 (1990), 1999-2009.

[8] D.E. Kashcheev, Compound Cox processes and option pricing, J. Math. Sci., 106 (2001), 2682-2690.

[9] S. Kotz, T.J. Kozubowski and K. Podgórski, The Laplace Distribution and Generalizations: A Revisit with Applications to Communications, Economics, Engineering, and Finance, Birkhäuser, Boston, 2001. 
[10] T.J. Kozubowski, M.M. Meerschaert and K. Podgórski, Fractional Laplace motion, Adv. Appl. Probab., 38 (2006), 451-464.

[11] T.J. Kozubowski and K. Podgórski, Distributional properties of the negative binomial Lévy process, preprint (2006).

[12] T.J. Kozubowski and S.T. Rachev, Univariate geometric stable laws, J. Comput. Anal. Appl., 1(2) (1999), 177-217.

[13] D.B. Madan, P. Carr and E.C. Chang, The variance gamma process and option pricing, European Finance Review, 2 (1998), 74-105.

[14] D.B. Madan and E. Seneta, The variance gamma (V.G.) model for share markets returns, J. Business, 63 (1990), 511-524.

[15] M.M. Meerschaert, T.J. Kozubowski, F.J. Molz and S. Lu, Fractional Laplace model for hydraulic conductivity, Geophysical Res. Lett., 31 (2004), L08501.

[16] R.N. Pillai, On Mittag-Leffler functions and related distributions, Ann. Inst. Statist. Math., 42(1) (1990), 157-161.

[17] J. Rosiński, Series representations for Lévy processes from the perspective of point processes, in: Lévy Processes: Theory and Applications, ed. O.E. Barndorff-Nielsen, T. Mikosch, and S.I. Resnick, pp. 401-415, Birkhaüser, Boston, 2001.

[18] S. Satheesh, Stability of Random Sums and Extremes, Ph.D. Thesis, Cochin University of Science and Technology, 2001.

[19] S. Satheesh, N.U. Naui and E. Sandhya, Stability of random sums, Stochastic Modeling and Applications, 5 (2002), 17-26.

[20] W. Schoutens, Lévy Processes in Finance: Pricing Financial Derivatives, John Wiley \& Sons, Chichester, 2003.

[21] D. Veneziano, Basic properties and characterization of stochastically self-similar processes in $\mathbb{R}^{d}$, Fractals, $7(1)$ (1999), 59-78.

Received: October 21, 2006 